\title{
Erratum to: Temperature scaling pattern dependence on representative concentration pathway emission scenarios
}

\section{A Letter}

Yasuhiro Ishizaki • Hideo Shiogama • Seita Emori •

Tokuta Yokohata • Toru Nozawa • Tomoo Ogura •

Manabu Abe - Masakazu Yoshimori • Kiyoshi Takahashi

Published online: 20 June 2014

(C) Springer Science+Business Media Dordrecht 2014

\section{Erratum to: Climatic Change (2012) 112:535-546 \\ DOI 10.1007/s10584-012-0430-8}

Unfortunately, there are several mistakes in the original publication of this article:

1. Figure 1c is missing. It is given here:

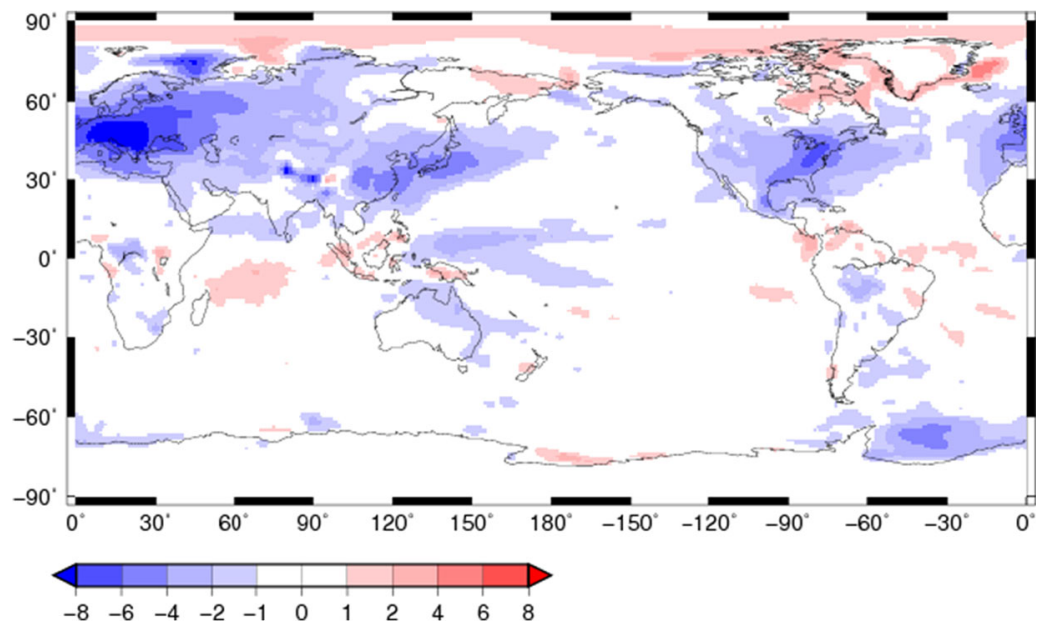

2. The last two graphs of Fig. $\mathbf{2}$ should have been labelled $\mathbf{c}$ and $\mathbf{d}$, not $\mathbf{a}$ and $\mathbf{b}$.

3. In the Acknowledgments section towards the end "(S-5-3)" should be "(S-5-1)".

The online version of the original article can be found at http://dx.doi.org/10.1007/s10584-012-0430-8.

Y. Ishizaki $(\bowtie) \cdot$ H. Shiogama $\cdot$ S. Emori $\cdot$ T. Yokohata $\cdot$ T. Nozawa $\cdot$ T. Ogura $\cdot$ M. Abe $\cdot$ K. Takahashi

National Institute for Environmental Studies, Tsukuba-City, Ibaraki, Japan

e-mail: ishizaki.yasuhiro@nies.go.jp

M. Yoshimori

Atmosphere and Ocean Research Institute, The University of Tokyo, Tokyo, Japan 